\title{
Assemblée générale de la Société des études robespierristes Samedi 18 mars 2000 Amphithéâtre Georges-Lefebvre
}

Marcel Dorigny

\section{(2) OpenEdition}

Journals

Édition électronique

URL : https://journals.openedition.org/ahrf/1835

DOI : 10.4000/ahrf.1835

ISSN : 1952-403X

Éditeur :

Armand Colin, Société des études robespierristes

Édition imprimée

Date de publication : 1 décembre 2000

Pagination : 181-187

ISSN : 0003-4436

Référence électronique

Marcel Dorigny, «Assemblée générale de la Société des études robespierristes Samedi 18 mars 2000 Amphithéâtre Georges-Lefebvre », Annales historiques de la Révolution française [En ligne], 322 | octobre-décembre 2000, mis en ligne le 22 mars 2006, consulté le 23 avril 2022. URL : http:// journals.openedition.org/ahrf/1835; DOI : https://doi.org/10.4000/ahrf.1835

Ce document a été généré automatiquement le 23 avril 2022

Tous droits réservés 


\title{
Assemblée générale de la Société des études robespierristes Samedi 18 mars 2000 Amphithéâtre Georges-Lefebvre
}

\author{
Marcel Dorigny
}

1 La Société des études robespierristes a tenu son assemblée générale annuelle ordinaire le samedi 18 mars 2000, à 9 heures, dans l'amphithéâtre Georges-Lefebvre, à la Sorbonne, sous la présidence de Michel Pertué.

3 M.MmeSerge Aberdam, Jean Bart, Yves Bénot, Jean-Paul Bertaud, Jacques Bernet, Philippe Bertholet, Serge Bianchi, Michel Biard, Marie-Françoise Blot, Bernard Bodinier, Nicole Bossut, Patrice Bret, FrançoiseBrunel, Irène Castells, Marie Cazein, Jean-Luc Chappey, ClaudeDurand-Coquard, Claude Coquard, Annie Crépin, Michaël Cufi, M.Cuttoli, Philippe Daumas, Jean Defranceschi, Robert Demeude, MarcelDorigny, Émile Ducoudray, Annie Duprat, Pascal Dupuy, BernardGainot, Annie Geffroy, Jean-Claude Halpern, Jean-PierreJessenne, Anne Jollet, Christine Le Bozec, Robert Leguillois, Christian Lescureux, Arezki Manseur, Hèlène Marle, Yoshito Matsuura, Raymonde Monnier, Jacques Najean, Michel Pertué, Danièle Pingué, Jean-Daniel Piquet, Thomas Pronier, Clément Riches, Michel Sisic, Eric Teyssier, Rachida Tlili-Sellaouti, Clément Riches, Marie-Jeanne Rossignol, Bernard Vinot, Claudine Wolikow.

4 Excusés :

5 M.Mme Marie-Claude Baron, Yannic Bosc, PhilippeBourdin, Malcom Crook, Florence Gauthier, Jacques Guilhaumou, Jean-Pierre Gross, Claude Mazauric, Matthias Middell, André Paris, Philippe Rahxon, AnnaMaria Rao, Alexandre Tchoudinov. Ont donné des pouvoirs :

7 Olivier Coquard, Geneviève Coulmy.

8 L'ordre du jour appelle l'examen des points suivants : 
Choix d'un bureau

L'assemblée choisit comme président et secrétaire ceux du conseil d'administration.

Désignation des commissaires aux comptes

En l'absence d'un règlement intérieur, qui doit être adopté au cours de la présente assemblée générale pour être ensuite soumis aux autorités de tutelle, le président propose à l'assemblée de reconduire, à titre provisoire, les deux commissaires aux comptes de l'an passé et de remplacer Maurice Genty, actuellement souffrant, par Nicole Bossut. Nicole Bossut et Jean-LucChappey sont élus à l'unanimité.

Lecture du rapport d'activité de l'année 1999

11 Marcel Dorigny donne lecture du rapport d'activité de l'année 1999, selon les termes qui avaient été adoptés par le conseil d'administration du 22 janvier dernier voir le procès verbal de cette réunion, ci-dessus.

12 La discussion est ouverte sur ce rapport :

13 Jacques Bernet demande qu'à l'avenir une meilleure information soit faite pour faire connaître la tenue des journées d'études. M.Dorigny rappelle que pour les journées des 10 et 11 décembre dernier, sur «Sciences et Révolution, le programme complet avait été publié dans le Bulletin de la Société française d'études du XvIIIe siècle paru en août, ainsi que dans le numéro3 des $A H R F$, paru début octobre. Il semble difficile, et surtout coûteux, de procéder à l'envoi d'une circulaire à tous les sociétaires, qui ferait double emploi avec la publication dans la revue. Cependant, pour la journée du 7 octobre 2000, consacrée à "La France et les Amériques au temps de Jefferson et Miranda», un affichage sera prévu.

14 Serge Aberdam fait remarquer que la publication du recueil d'articles sur les questions agraires n'est pas d'une perfection absolue il lui est répondu que, outre une regrettable erreur dans la coupure d'un texte, la qualité de la réimpression est liée à celle des textes originaux, lesquels sont d'une inégale lisibilité, notamment ceux extraits des AHRF des années 1920 et 1950.

Lecture du rapport financier et approbation des comptes de l'exercice clos

Claude Coquard donne lecture du rapport financier et des comptes de l'exercice 1999, qui ont été distribués aux membres présents après avoir examiné les documents financiers le lundi 13 mars précédent, les deux commissaires aux comptes proposent de donner quitus au trésorier. Après un court débat sur quelques points de détail, l'assemblée générale vote le quitus à l'unanimité.

Voir les documents en annexe du procès verbal du conseil d'administration du 22 janvier 2000, dans le n 3-2000 des AHRF.

Programme d'action pour l'année 2000

17 Marcel Dorigny donne lecture du programme prévu pour l'année en cours, adopté par le Conseil d'administration du 22 janvier dernier voir ci-dessus.

18 À l'issue de cette présentation, Jean-Paul Bertaud propose que la Société des études robespierristes prépare dès maintenant un colloque international consacré au bilan du Consulat et de l'Empire, qui pourrait se tenir dans deux ou trois ans. M. Pertué et M.Dorigny informent l'assemblée qu'une réflexion est en cours au sein du bureau de la Société sur ce sujet et que la proposition de Jean-Paul Bertaud est la bienvenue un prochain Conseil d'administration examinera un projet de colloque et définira les partenariats nécessaires à sa réalisation. 
19 Serge Aberdam propose que la réimpression annoncée de la brochure sur les utopies, publiée en 1977 par la Société et épuisée depuis longtemps, soit mise à jour, notamment par l'adjonction d'un supplément au répertoire des utopies. Le président retient cette proposition, à condition qu'une telle adjonction soit scientifiquement fondée, les comptes rendus qui avaient été publiés lors de la première édition de ce travail ayant davantage souligné l'élargissement par les auteurs, I. Hartig et A. Soboul, la notion d'utopie que de graves lacunes.

Jean-Pierre Jessenne demande que les futures journées d'études fassent l'objet d'un débat approfondi afin de les insérer dans une démarche d'ensemble de la Société. Le président lui répond que ce point sera inscrit à l'ordre du jour du prochain conseil d'administration.

21 Après ces quelques échanges, le programme proposé par le conseil d'administration est approuvé à l'unanimité par l'assemblée générale.

Vote du budget prévisionnel pour l'année 2000

22 Claude Coquard, trésorier, lit et commente le projet de budget distribué aux sociétaires en début de séance.

23 Voir le budget prévisionnel en annexe du procès verbal du conseil d'administration du 22 janvier 2000, dans le ${ }^{\circ}$ 3-2000 des AHRF.

Après cette présentation, le président prend la parole pour souligner que les finances de la Société sont saines et faire deux observations :

-les coûts de production de la revue, à qualité inchangée, ont été réduits de $25 \%$, soit une économie de 60000 francs par an.

-si les réserves de la Société sont estimées à la baisse au 31décembre 2000, à hauteur de 245000 francs, cela tient au fait que les dépenses d'édition sont cette année très importantes (report de l'exercice précédent et charges exceptionnelles de l'index) et que des subventions encore incertaines, mais très probables, n'ont pas été indiquées en recette. Par ailleurs, les frais de publications des actes des journées d'études seront vraisemblablement reportés pour partie sur l'exercice 2001.

27 Après un court débat, notamment consacré aux réserves financières de la Société et à la possibilité de placement en SICAV, le projet de budget est adopté à l'unanimité des présents.

Adoption du règlement intérieur

Le projet de règlement intérieur adopté par le conseil d'administration du 22 janvier, à l'unanimité moins une abstention, a été envoyé aux sociétaires par le même courrier que la convocation à l'assemblée générale. Lecture en est faite, article par article, par M.Pertué. Après lecture de l'article 2 du titre II, consacré au mode de renouvellement du comité de rédaction de la revue, SergeAberdam demande la parole et propose une modification de la rédaction initiale afin de permettre le renouvellement par moitié du comité de rédaction à chaque renouvellement partiel du conseil d'administration, tous les trois ans, au lieu d'un renouvellement complet du comité de rédaction tous les six ans seulement, comme c'est le cas du directeur de la revue. M.Pertué rappelle que le mandat du directeur étant de six années, il est, à ses yeux, préférable de désigner le comité de rédaction pour la même durée. Après un échange de points de vues, au cours duquel Claude Coquard soutient la proposition de Serge Aberdam, celle-ci est mise aux voix. Par 16 voix contre 14 et 7 absentions, le reste de l'assemblée ne prenant pas part au vote, la nouvelle rédaction de l'article 2 du titre II est adoptée: «Le comité de 
rédaction est renouvelé par moitié après chaque renouvellement partiel du conseil d'administration. »

Sur proposition de Michel Biard, et après discussion au sein de l'assemblée, l'article 1 $\mathrm{du}$ titre II du règlement intérieur est modifié ainsi « Le directeur de la revue réunit le comité de rédaction au moins une fois par semestre.»

Les autres articles du règlement intérieur sont approuvés à l'unanimité.

Voir le texte définitivement adopté en annexe dans le n³-2000 des AHRF.

Information de l'assemblée sur la composition du comité scientifique

La composition du comité scientifique a été approuvée par le conseil d'administration $\mathrm{du} 10$ juin dernier voir la liste en troisième page de la revue.

Après cette lecture, Irène Castells rappelle que deux collègues espagnols, Alberto Gil Novales et Joseph Fontana n'avaient pu être sollicités à temps, mais que depuis ils ont donné leur accord pour participer au comité scientifique. Il est décidé que le prochain conseil d'administration prendra en compte cette modification de la composition du comité scientifique.

Admission de nouveaux membres

Conformément à l'article 3 du titre I des statuts, le président donne lecture des noms, prénoms, qualités et parrains respectifs des 31 personnes qui souhaitent adhérer à la Société des études robespierristes :

Pierre-Yves Beaurepaire, maître de conférences (A. Crépin et M.Dorigny), Yves Bénot, retraité (M. Dorigny et B. Gainot), Jean-Charles Benzaken, professeur de collège (M. Dorigny et M. Vovelle), Philippe Bertholet, professeur de lycée (C. Coquard et M. Dorigny), Julien Boudon, chargé de TD et étudiant (A. Duprat et R. Monnier), Justine Chauvel, étudiante (M. Dorigny et B. Vinot), Sophie Coupry, étudiante (M. Dorigny et B. Gainot), Michaël Cufi, étudiant (A. Crépin et J-P. Jessenne), Françoise Dauphin, retraitée (S. Bianchi et A. Crépin), Renata De Lorenzo, professeur des universités (Ph. Bourdin et A.M. Rao), Robert Demeude, retraité (S. Bianchi et B. Gainot), André Destouches, professeur de lycée (A. Crépin et B. Gainot), Ouzi Elyada, professeur des Universités (M. Dorigny et A.Duprat), Patrick Garcia, maître de conférences (M. Dorigny et B.Gainot), Eric Gojosso, maître de conférences (M. Dorigny et M. Pertué), Jouda Guetata, étudiante (M. Dorigny et F. Gauthier), François Jacob, maître de conférences (M. Dorigny et B. Gainot), Isabelle Laboulais-Lesage, maître de conférences (C. Mazauric et Ch. Le Bozec), EricaMannucci (M. Dorigny et R. Monnier), Lucie Maquerlot, professeur de collège (M. Dorigny et C. Mazauric), Jean-Clément Martin, professeur des Universités (M. Mazauric et M. Pertué), Claire Mauvais, retraitée (S.Bianchi et A. Crépin), Marie-Hélène Pardoen, étudiante (Ph. Bourdin et A. Crépin), Christophe Pouzieux, ATER (M. Dorigny et M. Pertué), Thomas Pronier, étudiant (M. Dorigny et B. Gainot), Frédéric Régent, professeur d'histoire (Fr. Brunel et J-P Bertaud), Marie-Jeanne Rossignol, professeur des universités (M. Dorigny et B. Gainot), Kôbô Seigan, maître de conférences (A. Crépin et B. Gainot), Michel Sicsic, professeur de lycée (M. Dorigny et B. Gainot), Ph. Tanchoux, allocataire de recherches (C.Coquard et M. Pertué), Éric Teyssier, PRAG (B. Bodinier et M.Dorigny).

L'assemblée les admet à l'unanimité comme membres de la Société.

Questions diverses

Jean-Paul Bertaud intervient pour demander qu'un prochain numéro de la revue publie un compte rendu argumenté du livre de PatriceGueniffey, La politique de la Terreur. Essai 
sur la violence révolutionnaire.1789-1794, paru aux Éditions Fayard Michel Biard intervient à son tour pour s'opposer à un tel compte rendu, en raison du caractère nonscientifique et largement polémique de l'ouvrage. Un bref débat s'ouvre sur cette question au cours duquel, notamment, Jacques Bernet, Serge Aberdam et Jean Defranceschi considèrent qu'une réponse scientifique est nécessaire. Le président propose que le comité de rédaction soit chargé, comme pour tous les livres reçus, de demander à l'un des sociétaires de préparer un compte rendu de ce livre, à paraître dans un prochain numéro de la revue.

Une motion est proposée au vote de l'assemblée générale par ClaudeMazauric, pour protester contre la récente réforme du Comité des travaux historiques et scientifiques, imposée par le Ministère de l'Enseignement supérieur et de la recherche. La Commission d'histoire de la Révolution française du CTHS, dite "Commission Jaurès, est désormais réunie à une partie de l'ancienne section d'histoire moderne et contemporaine, au sein d'une nouvelle section dite « d'histoire du monde moderne, de la Révolution française et des révolutions. Lecture faite du projet de motion, un débat s'engage, au cours duquel Jean Bart, président de la Commission d'Histoire de la Révolution française, explique qu'il est impropre de parler de "suppression mais de fusion et d'élargissement de la sphère de compétence de l'ancienne Commission puisque les révolutions du xixe siècle y sont désormais incluses il souligne, en revanche, le peu de concertation préalable et le caractère autoritaire de la décision prise par l'autorité de tutelle Jacques Bernet et Claudine Wolikow interviennent dans le même sens et soulignent l'impossibilité de maintenir durablement une spécialisation étroitement enfermée dans la période 1789-1799 Jean-Paul Bertaud, quant à lui, dénonce avec force cette disparition de l'autonomie de la "Commission Jaurès au sein du CTHS et y voit la perte d'un symbole fort des études révolutionnaires.

39 Au terme de ces échanges de vues, la motion est mise aux voix. Le scrutin donne le résultat suivant : 3 refus de vote ; 8 absentions et 37 voix pour. La motion, dont le texte suit, est donc adoptée :

41 "Réunie en assemblée générale à la Sorbonne le 18 mars 2000, la Société des études robespierristes (reconnue d'utilité publique depuis 1935) proteste contre la suppression de la spécificité de la "Commission d'histoire de la Révolution française au sein du comité des travaux historiques et scientifiques.

42 La Société rappelle que cette commission, héritière de celle qu'avait instituée en 1903 la Chambre des députés sur la proposition de Jean Jaurès, a depuis regroupé les meilleurs spécialistes français de l'histoire de la Révolution, d'Aulard à Mathiez et Lefebvre, de Labrousse à Reinhard, Soboul, Godechot et Suratteau, pour n'évoquer que les disparus et souligne que, par son activité, elle a contribué, notamment à l'occasion du bicentenaire de 1989, à l'approfondissement et au renouvellement de l'historiographie de la Révolution.

La Société prie Monsieur le Ministre de l'Éducation nationale de bien vouloir modifier son arrêté du 11 février, de telle manière que cette commission, que chacun connait sous le nom de "Commission Jaurès, puisse poursuivre son irremplaçable cuvre scientifique en préservant son autonomie et sa singularité dans l'esprit de ce qui fut décidé en 1983 et 1986. »

44 Le président est chargé de la transmettre aux autorités de tutelle Ministère de l'Enseignement supérieur et de la recherche et Ministère de l'intérieur. 
Jean-Paul Bertaud demande si le remboursement des frais de déplacement des membres étrangers du comité de rédaction est envisagé par la Société. Le président répond que l'importance des frais qui seraient engagés pour le déplacement deux à quatre fois par an des 14 membres concernés rend impossible le remboursement demandé.

L'ordre du jour étant épuisé, la séance est levée à 11 h 45.

La séance a été prolongée par une journée d'étude portant sur thème suivant :

«Révolution agraire ou maintien de la plantation après les abolitions révolutionnaires de l'esclavage?» des révolutions antiesclavagistes, de 1791 à 1848, deux communications ont été entendues:

Gérard Barthélémy (Anthropologie et histoire. Université de Port-au-Prince) « De SaintDomingue à Haïti une révolution paysanne ?» Puis de $14 \mathrm{~h} 30$ à $17 \mathrm{~h}$ :

Chivallon (Chargée de recherche, TIDE-CNRS, M.S.H. d'Aquitaine): «La Martinique après 1848 l'occupation des mornes par les nouveaux libres " Les deux communications, devant un public élargi par la présence des membres de l'Association pour l'Étude de la Colonisation européenne, co-organisatrice de la journée d'étude, ont été suivies d'un débat.

\section{AUTEUR}

MARCEL DORIGNY

Le secrétaire général 